\title{
Rehabilitation in Subjects with Chronic non-specific Low Back Pain with Sacroiliac Joint Origin: Protocol for a Systematic Review
}

\author{
T. Shahmahmoodi1,2, S. Izadi Laybidy ${ }^{1,2}$, A. Rahimi ${ }^{3}$, F. Bokaee1, Z. S. Rezaeian ${ }^{1}$ \\ 1 Musculoskeletal Research Center and Department of Physical Therapy, Faculty of Rehabilitation Sciences, \\ Isfahan University of Medical Sciences, Isfahan, Iran \\ 2 Student Research Committee of Rehabilitation Students (Treata), Faculty of Rehabilitation Sciences, Isfahan \\ University of Medical Sciences, Isfahan, Iran \\ 3 Health Information Research Center, Head of Clinical Informationist Research Group, Faculty of Management \\ and Medical Information Sciences, Isfahan University of Medical Sciences, Isfahan, Iran
}

\section{CORRESPONDING AUTHOR: \\ Zahra Sadat Rezaeian \\ Musculoskeletal Research Center \\ Rehabilitation Sciences Research Institute \\ Department of Physical Therapy \\ Faculty of Rehabilitation Sciences \\ Isfahan University of Medical Sciences \\ Hezar Jerib Avenue \\ Isfahan, Iran 8174673461 \\ E-mail: zrezaeian@rehab.mui.ac.ir}

DOI:

10.32098/mltj.04.2020.21

LEVEL OF EVIDENCE: 1A

\begin{abstract}
SUMMARY
Introduction. This systematic review protocol aims to evaluate the effect of rehabilitation for chronic non-specific low back pain with sacroiliac joint origin.

Methods. Search will be done in Pubmed, ISI Web of Science, Scopus, Clinical Key, Science Direct, Medline, Embase, PEDro, ProQuest, the Cochrane Library, PROSPERO, the MOH Thesis, MOH Articles, Magiran, and SID. Google Scholar search engine will also be used. All types of Clinical Trials, Cohort, Case-controls, Cross-sectionals, Observational Descriptive, Case Report, Case Series, Ecological Studies, Systematic Reviews, thesis and dissertation in English and Persian published prior to September 2019 will be included. The articles recruiting 18 to 60 years old will be included. Considering PICO, the finally retrieved articles will be assessed qualitatively by CONSORT, STROBE, PEDro, NIH and CASP checklists. Changes in pain and function will be favorable.

Dissemination. The protocol presented in present paper will be used to summarize and qualify present literatures on conservative therapy for chronic non-specific low back pain with sacroiliac joint origin.
\end{abstract}

\section{KEY WORDS}

Rehabilitation; phisycal therapy; pain; function; non-specific chronic low back pain; sacroiliac joint dysfunction.

\section{INTRODUCTION}

Today, Low Back Pain (LBP) has become one the most common musculoskeletal disorder in the societies with average global prevalence of $38.5 \%(1,2)$. It is hypothesized that the $15-30 \%$ of Chronic Non-specific Low Back Pain (CNLBP) have sacroiliac joint origin (3-5). They are two main approaches dealing with sacroiliac joint impairment (6, 7). Non-invasive treatments such as physiotherapy (8-11) are the forefront of treatment in these cases (12-14). If not helpful, invasive approaches including fixation or fusion may be prescribed (15).
Since physiotherapy interventions as a non-invasive technique seems to be effective in treating chronic LBP (16), the aim of this study is to design a systematic review study seeking and comparing the effects of various interventions in this category on the pain and function of subjects that suffer from CNLBP with sacroiliac joint origin; the results will be of clinical value to determine the effectiveness of each method for this particular subgroup of LBP. If enough paper retrieved, the comparison can be made and a comprehensive therapeutic physical therapy plan may be suggested. 


\section{OBJECTIVES}

To determine the effect of rehabilitation interventions on the pain and function in individuals suffering from CNLBP with sacroiliac joint origin.

\section{MATERIALS AND METHODS}

\section{Trial eligibility criteria}

Strict inclusion/exclusion criteria have been introduced in order to precisely protect search strategies and PICOs. The criteria are summarized below.

\section{Study types}

All study types, except Qualitative Studies, and Narrative Reviews, i.e. Clinical Trials, Cohort, Case-controls, Cross-sectionals, Observational Descriptive, Case Report, Case Series, Ecological Studies, Systematic Reviews and thesis and dissertation will be included.

\section{Participants}

People between 18-60 with no regard to gender and ethnicity who suffer from non-specific CLBP of sacroiliac joint origin and received conservative/rehabilitative interventions. The study will be approved for more detailed analysis if the participants suffered from back pain not less than three months with the signs of SIJ involvement. Studies targeting nonhuman samples, professional athletes, subjects with acute LBP, symptoms persisted for less than three months will be excluded. LBPs of specified origin like inflammatory diseases, spondylo-arthropathies, disk hernia, spinal canal/foraminal stenosis, visceral pains, fractures and trauma, those with referral or radicular symptoms, studies on pregnant women, children (under 18 years) and elderly (over 60 years) will be excluded.

\section{Interventions}

At least one of the study groups has to undertake a rehabilitative or conservative intervention including:

- electrotherapy modalities: electrical stimulation currents (Transcutaneous Electrical Nerve Stimulation (TENS), Interferential (IF), Diadynamic, High-voltage, Russian currents, Faradic...), LASERs, ultrasound, shockwave, tecar, magnet, shortwave and microwave diathermy, infra-red radiation, hot packs, cold packs...;

- manual techniques: mobilization, manipulation, Muscle Energy Techniques (MET), soft tissue release, massage techniques, Instrumented Assisted Soft Tissue Manipulation (IASTM), visceral manipulation...;

- exercise therapy: any type of exercise including the proprioceptive neuromuscular facilitation (PNF) approaches;
- taping: Kinesio Taping ${ }^{\circledR}$, McConnell \& Mulligan, elastic bandages, Prophylactic athletic taping, non-medicated taping;

- needling: dry needling, acupuncture, electro-acupuncture; - orthosis.

\section{Comparators}

Studies that compare the effect of aforementioned interventions with a control group (without treatment), sham group (placebo treatment), healthy group (of matched healthy subjects) or those comparing two or more interventions will be included.

\section{Outcome measures}

Studies will be included that the experimental (case) group and the control group were established, and the related monitoring data were introduced. Pain and function will be the primary outcome measures if are reported by valid scale or devices. Pain will be assessed by the Numerical Rating Scale (NRS), Visual Analogue Scale (VAS), Pressure Pain Threshold (PPT), McGill Pain Questionnaire, pain provocation tests. Function will be measured by Roland-Morris Disability Questionnaire, Oswestry Disability Index, or clinical/functional tests like active single leg raise, reverse single leg raise. Other tools may be also considered according to the included studies.

Two classification variables, continuous variables and variance test should be administered. Within-(pre-post) and between-group measures will be analyzed for clinical trials. For cohorts and case-controls odds ratio will be of interest. Effect size and confidence intervals will be of value in all types included studies.

Additional outcome measures will may be considered upon progression of the study. Some of anticipated secondary outcome measures are: anthropometric data (weight, height, BMI), psycho-social and cultural data (literacy level, marital status, economical class), comorbidities (diabetes, cardiac or pulmonary disorders, smoking, alcohol consumption).

\section{Search methods to identify studies}

Articles will be accessed from international (Pubmed, ISI Web of Science, Scopus, Clinical Key, Science Direct, Medline, Embase, PEDro, ProQuest, the Cochrane Library, PROSPERO) and national (MOH Thesis, $\mathrm{MOH}$ Articles, Magiran, and SID) databases. Google Scholar search engine will also be searched.

Although narrative reviews and qualitative studies will not be targeted in current project, their references will be checked through Cross Reference. The main key words will be rehabilitation, conservative, physical therapy, pain, func- 
tion, non-specific chronic low back pain, sacroiliac that will be updated with study progression. The search strategy for accessing articles will be like the following search query and covers PICO.

Nonspecific AND chronic AND ("low back pain" OR (low AND back AND pain) OR "back ache") AND ("sacroiliac joint*" OR (sacroiliac AND joint) OR "sacroiliac*”) AND ("electric stimulation*" OR (electric AND stimulation) OR "electrical stimulation*") AND (TENS OR "Transcutaneous Electrical Nerve Stimulation" OR Interferential OR Diadynamic OR High Voltage OR Russian OR Faradic OR LASERs OR ultrasound OR shockwave OR tecar OR magnet OR shortwave OR microwave OR diathermy OR infra-red OR hot pack OR cold packs OR ("manual techniques*" OR (manual AND technique)) OR mobilization OR manipulation OR ("muscle energy" OR (muscle AND energy)) OR ("soft tissue release ${ }^{* ”}$ OR ("soft tissue *" $^{*}$ AND release) OR ("visceral manipulation" OR (visceral AND manipulation)) OR ("instrumented assisted soft tissue manipulation*" OR ("instrumented assisted*" AND "soft tissue manipulation*") OR exercise OR ("proprioceptive neuromuscular facilitation*" OR (proprioceptive AND neuromuscular AND facilitation) OR taping OR needling OR acupuncture OR orthosis) AND ("control group*" OR ((placebo or unrealistic) AND (treatment OR therapy*)) AND Function*) in TITLE/SUMMARY/KEY WORDS.

P: nonspecific AND chronic AND "low back pain" OR (low AND back AND pain) OR "back ache").

I: ("electric stimulation*" OR (electric AND stimulation) OR "electrical stimulation*") AND (TENS OR "Transcutaneous Electrical Nerve Stimulation" OR Interferential OR Diadynamic OR High Voltage OR Russian OR Faradic OR LASERs OR ultrasound OR shockwave OR tecar OR magnet OR shortwave OR microwave OR diathermy OR infra-red OR hot pack OR cold packs OR ("manual techniques*" OR (manual AND technique)) OR mobilization OR manipulation OR ("muscle energy*" OR (muscle AND energy)) OR ("soft tissue release*" OR ("soft tissue*" AND release) OR ("visceral manipulation" OR (visceral AND manipulation)) OR ("instrumented assisted soft tissue manipulation*" OR ("instrumented assisted*" AND "soft tissue manipulation"”) OR exercise OR ("proprioceptive neuromuscular facilitation*" OR (proprioceptive AND neuromuscular AND facilitation) OR taping OR needling OR acupuncture OR orthosis).

C: "control group*" OR ((placebo or unrealistic) AND (treatment OR therapy)).

O: Therapy OR Treatment OR function*.

The research process will be conducted independently by two researchers (SIL and TSM) and their results will be compared each week. For all included articles, the search in the reference list (Hand Search) will also be performed. If the full text of the article is not found, the researchers will email the authors or the editor of the journal three times. If the reply was not satisfying, that article will be excluded. To find grey sources, special search in their related databases including registries of clinical trials (i.e. http://www.irct.ir/), http://www.trialscentral.com/, http://www.proquest.com/, http://www.gateway.com/worldwide/ will be done.

\section{Study selection}

Any article published before the end of September 2019 (Shahrivar 9 th 1398 Persian Calendar) will be potentially suitable. The search will be extended three years before the publication of the first article in the field of each intervention type. Search results and Reference lists will be transferred into the citation manager software. Duplicates and those marked as irrelevant will be ignored by screening titles and summaries. The full text of the remaining articles will be reviewed in detail. Under supervision of peers, i.e. ZSR, $\mathrm{FB}$ and $\mathrm{AR}$, the whole procedure will run by two researchers (SIL and TSM) who are blind to each other's work. Any ambiguity or controversy will be discussed in consensus.

Figure 1 summarizes the study selection flow according to the PRISMA flow diagram.

\section{Data extraction}

The databases mentioned previously will be searched. To determine the inter-rater agreement, screening of PubMed title/summaries will be done independently by two researchers (SIL and TSM) as supervised by ZSR, AR and FB. The reference list in excluded articles types (qualitative studies and narrative reviews) will be checked by Cross Referencing. The search line will be revised and key words will be updated as the project progresses. Key words from included studies will be merged in the search line. The references list of all accepted studies will be checked using Hand Search strategy. If the researchers did not access the full text of any article, the corresponding/first author or the editorial board of the publishing journal will be emailed thrice. If not effective, the article will be excluded.

The screening of the title/summaries will be performed independently by SIL and TSM and duplicated articles or unrelated ones will be excluded. They also will report the number of the articles retrieved from each database in a flowchart. The whole search results will be transferred into a citation manager. The screening will be start over every three months for validating fast exclusions.

The research team (SIL, ZSR, FB, AR and TSM) will criticize the included studies according to their full-text inde- 


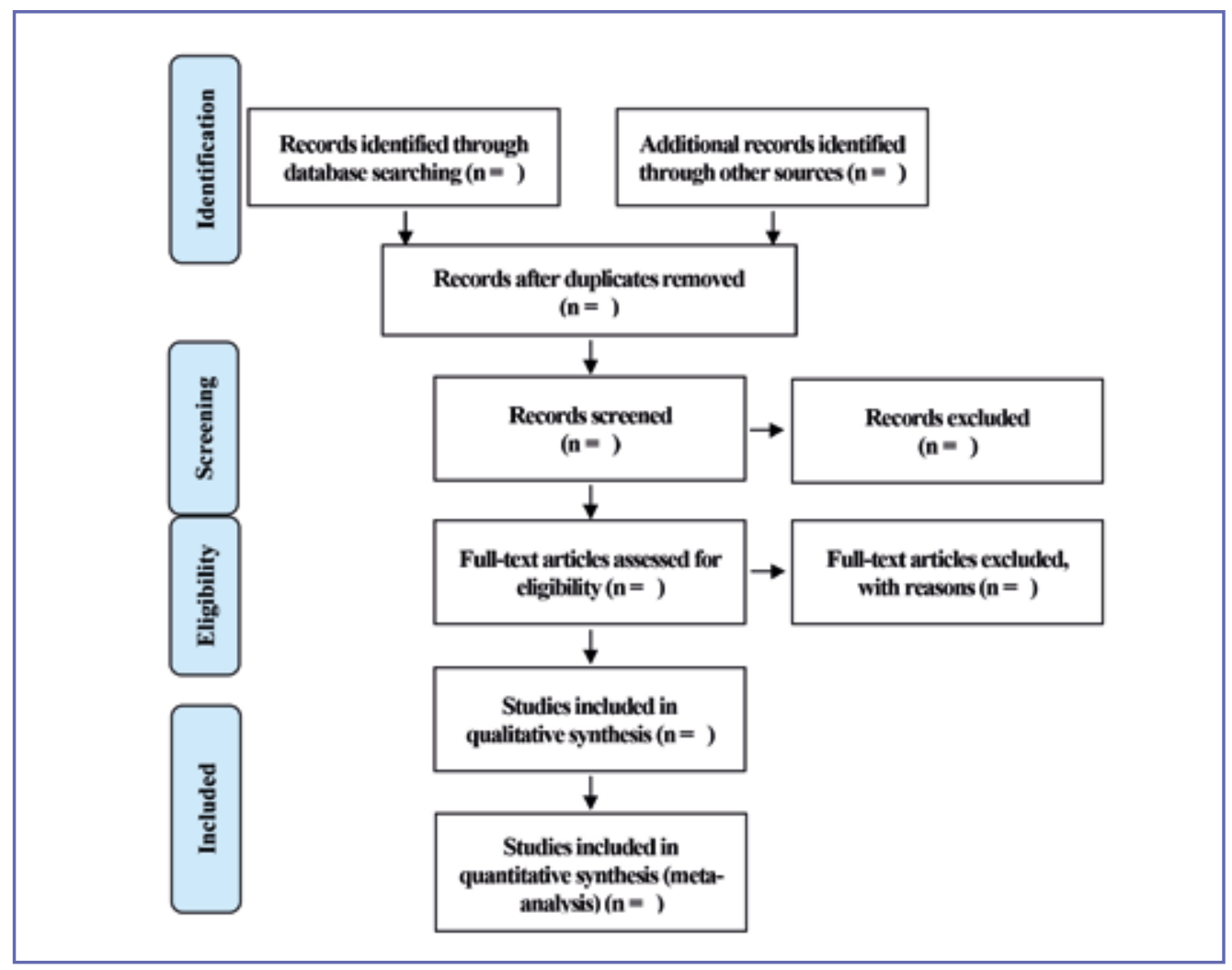

Figure 1. PRISMA 2009 Flow diagram (17) of the articles selection process.

pendently. Final decision on whether a study should be accepted for quality and quantity analysis will be made according to inclusion/exclusion criteria in a consensus. Reasons for the exclusion will be reported.

Data from the approved full-texts will be categorized in an Excel sheet (data extraction) according to publication indices (the author(s), title, publication year, journal, country), participants, study design, sample size, randomization, allocation concealment, blinding, intervention, control intervention, main outcomes, adverse effects, follow-up, withdrawals and results. If needed, more information will be requested from original authors. PI (ZSR) supervises the procedure. Any ambiguity or controversy in any phase will be illuminated in the consensus.

\section{Quality assessment}

Supervised by PI (ZSR), each approved article will be qualified in expert consensus with regard to its design by Consort, STROBE, PEDro, CASP and NHLBI checklists. Every checklist will be scored. Articles that get at least $50 \%$ of total score of one checklist will be approved quantitative analysis. The articles' quality will be considered as high $(75 \%)$, medium $(50-75 \%)$, low $(25-50 \%)$, poor $(<25 \%)$ based on the scores they gained by each single checklist. Any ranking dissimilarities will be discussed in expert consensus.

PEDro is an 11-item scale for clinical trials with "plus" (well addressed items) or "minus" (not-localized item) marks (18). In addition, as the identical, internationally accepted standard for qualification of clinical trials (19), the CONSORT checklist will also be used in present work. For more detailed assessment, the appropriate CONSORT extension may also be used (20).

The STROBE designed separate evaluation checklists to qualify case-control studies, cohort and other designs of studies $(21,22)$. On the other hand, some researchers believe that the STROBE checklists are not for formal quality assessment like the procedure required in a systematic review and recommend Study Quality Assessment Tools proposed by National Heart, Lung, and Blood Institute (NHLBI) instead (23). Both STROBE and NHLBI checklists were be administered in present study.

CASP also introduced specific checklist for each study designs (24) and will be used for determining the evidence ranking. For inclusive qualification of all article types, TIDieR checklist will be administered beside the main checklist. 


\section{Measure of Treatment Effects}

Mean Difference (MD) with a 95\% CI will be the format of choice for reporting continuous outcomes (like pain scales). If other forms of reports are present in articles, they will be covert into MD. For dichotomous or binary data (i.e. adverse events), a risk ratio (RR) with a $95 \%$ CI will be calculated.

\section{Missing data}

If there was a potential of missing data, the original research teams will be contacted. If they do not reply properly, only available data will be analyzed.

\section{Statistical Methods}

\section{Data synthesis}

If the number of homogenous studies was sufficient, data synthesis will be conducted using Stata software 8.0 (Stata Corporation, College Station, TX). If possible, meta-analysis will be considered using RevMan (Review Manager Software, Version 5.3; Copenhagen: The Nordic Cochrane Centre, The Cochrane Collaboration, 2014, The Cochrane Collaboration, Oxford, England). The RR and MD with the 95\% CI will be determined for dichotomous and continuous data respectively. Heterogeneity assessment will be carried out using the Q test and the $\mathrm{I}^{2}$ index. If $\mathrm{I}^{2} \leq 50 \%$, fixed-effects model will be used for calculating the RR and MD; the Mantel-Haenszel random-effects model will be applied, and aggregate participant data will be used. Nonetheless, the random-effects model will be of choice. If quantitative synthesis is not applicable, the results will be discussed descriptively. The procedure will be performed by two researchers (SIL and TSM) independently. Again, any disagreements will be resolved through consensus.

\section{Assessment of heterogeneity}

For calculating the heterogeneity, the $\mathrm{I}^{2}$ and $\chi^{2}$ tests will be used. In $\mathrm{I}^{2}$ analysis the cut off will be set as $50 \%$. If $\mathrm{I}^{2}>50 \%$, subgroup analysis will be run to highlight the potential factors.

\section{Subgroup and sensitivity analysis}

In order to determine the heterogeneity among included studies, subgroup analysis that categorizes each intervention mode according to its frequency and/or timing, type of control, countries and different outcomes may be considered. Then, if the heterogeneity persists or if studies with incomplete results were included, the sensitivity analysis will be done with omitting low quality articles. The meta-analysis will be developed again and the results of these two meta-analyses will be matched and discussed in terms of the sample size, strength of evidence and influence on the pooled effect size.

\section{Assessment of reporting biases}

Risk of bias will be assessed by two reviewers (SIL and TSM) using the Cochrane Collaboration's tool. Disagreements will be resolved in consensus. Reaching appropriate number of studies for qualitative analysis (at least 10 per intervention), funnel plots will be developed for analyzing the publication bias. Besides, the effect of possible selective reporting, reporting deviations from the original protocols, effect of protocol compliance and adherence will be tracked.

\section{DISCUSSION}

This systematic review will provide a comprehensive search to retrieve all existing evidences concerning the effects of physiotherapy interventions in CNLBP with sacroiliac joint origin. The inclusion and exclusion criteria provide a reasonable base to assure that these effects will be discussable in temporal spectrum from immediate to very long term effects upon the time intervals of follow ups in the included studies. The main reason for conducting this review was to summarize clinical value of physiotherapy in treatment of these subjects with regard to evidence hierarchy and indicate their strengths. For best internal validity, various checklists will be administered for each study design and articles scoring will be scheduled in a peers' consensus.

The study will provide data for developing rehabilitative guidelines, apprise insurance coverage and standard protocols of physical therapy planning. According to the retrieved articles, cost-effectiveness and best practice of various physiotherapy intervention may be judged. However, it should be kept in mind that the main challenge in rehabilitation of CNLBP with sacroiliac origin is the accuracy of diagnosis that is not easily confirmed based on clinical examination alone. This fact needed to be appropriately dealt with in the original studies included in the review. We will try to collect all existing studies in this field covering all study designs and all physiotherapy interventions subheadings. The review results will also highlight the existing research and clinical gaps to conduct future researches.

As the search has been started right now, it seems that there is not any study available concerning some interventions. In addition, meta-analysis will be applicable only if the retrieved articles were not heterogeneous.

\section{ETHICS}

The study has been funded and ethically approved by Isfahan University of Medical Sciences (Ethics Code: IR.MUI. REC.1397.335) as a part of a thesis for Master's Degree in Physical Therapy by Taraneh Shahmahmoodi (Registration 
code: 297122$)$. The sponsor has no role in data collection, analysis of the data and drafting the manuscript. The study meets the ethical standards of the journal of Muscle, Ligament, and Tendon Journal (25).

\section{ACKNOWLEDGMENTS}

This study will be developed with the financial support and ethically approval by Isfahan University of Medical Sciences (Ethics Code: IR.MUI.REC.1397.335) as a part of a thesis

\section{REFERENCES}

1. Balague F, Mannion AF, Pellise F, Cedraschi C. Non-specific low back pain. Lancet 2012;379:482-91.

2. Hoy D, Bain C, Williams G, et al. A systematic review of the global prevalence of low back pain. Arthritis Rheum 2012;64:2028-37.

3. Zaidi HA, Montoure AJ, Dickman CA. Surgical and clinical efficacy of sacroiliac joint fusion: a systematic review of the literature. J Neurosurg Spine 2015;23:59-66.

4. Van der Wurff P, Meyne W, Hagmeijer RHM. Clinical tests of the sacroiliac joint-A systematic methodological review. Part 2: Validity. Man Ther 2000;5:89-96.

5. Adhia DB, Tumilty S, Mani R, Milosavljevic S, Bussey MD. Can hip abduction and external rotation discriminate sacroiliac joint pain? Man Ther 2016;21:191-7.

6. Rashbaum RF, Ohnmeiss DD, Lindley EM, Kitchel SH, Patel VV. Sacroiliac Joint Pain and Its Treatment. Clin Spine Surg 2016;29:42-8.

7. Cohen SP. Sacroiliac joint pain: A comprehensive review of anatomy, diagnosis, and treatment. Anesth Analg 2005;101:1440-53.

8. Kamali F, Shokri E. The effect of two manipulative therapy techniques and their outcome in patients with sacroiliac joint syndrome. J Bodyw Mov Ther 2012;16:29-35.

9. Dhinkaran M, Sareen A, Arora T. Comparative analysis of Muscle Energy Technique and conventional physiotherapy in treatment of sacroiliac joint dysfunction. Indian J Physiother Occup Ther 2011;5:127-30.

10. Bradley MH, Rawlins A, Brinker CA. Physical Therapy Treatment of Pelvic Pain. Phys Med Rehabil Clin N Am 2017;28:589-601.

11. Beales D, Hope JB, Hoff TS, Sandvik H, Wergeland O, Fary $\mathrm{R}$. Current practice in management of pelvic girdle pain amongst physiotherapists in Norway and Australia. Man Ther 2015;20:109-16.

12. Richardson CA, Snijders CJ, Hides JA, Damen L, Pas MS, Storm J. The relation between the transversus abdominis muscles, sacroiliac joint mechanics, and low back pain. Spine 2002;27:399-405.

13. Polly DW, Swofford J, Whang PG, et al. Two-year outcomes from a randomized controlled trial of minimally invasive sacroiliac joint fusion vs. non-surgical management for sacroiliac joint dysfunction. Inter J spine surg 2016;10:1-22. for Master's Degree in Physical Therapy by Taraneh Shahmahmoodi (Registration code: 297122). The protocol has been registered in International Prospective Register of Systematic Reviews (CRD42020121383). The sponsor will not play a role in data collection, analysis of the data and drafting the manuscript.

\section{CONFLICT OF INTERESTS}

The authors declare that they have no conflict of interests.

14. Monticone M, Barbarino A, Testi C, Arzano S, Moschi A, Negrini S. Symptomatic efficacy of stabilizing treatment versus laser therapy for sub-acute low back pain with positive tests for sacroiliac dysfunction: a randomised clinical controlled trial with 1 year follow-up. Eura Medicophys 2004;40:263-8.

15. Lingutla KK, Pollock R, Ahuja S. Sacroiliac joint fusion for low back pain: a systematic review and meta-analysis. Eur Spine J 2016;25:1924-31.

16. Qaseem A, Wilt TJ, McLean RM, Forciea MA. Noninvasive Treatments for Acute, Subacute, and Chronic Low Back Pain: A Clinical Practice Guideline From the American College of Physicians. Ann Intern Med 2017;166:514-30.

17. Moher D, Liberati A, Tetzlaff J, Altman DG. Preferred reporting items for systematic reviews and meta-analyses: the PRISMA statement. J Clin Epidemiol 2009;62:1006-12.

18. Maher CG, Sherrington C, Herbert RD, Moseley AM, Elkins M. Reliability of the PEDro scale for rating quality of randomized controlled trials. Phys Ther 2003;83:713-21.

19. Moher D, Hopewell S, Schulz KF, et al. CONSORT 2010 explanation and elaboration: updated guidelines for reporting parallel group randomised trials. BMJ 2010;63:28-55.

20. CONSORT group. www.consort-statement.org/extensions. 2019.

21. Vandenbroucke JP, von EE, Altman DG, et al. Strengthening the Reporting of Observational Studies in Epidemiology (STROBE): explanation and elaboration. Int J Surg 2014;12:1500-24.

22. Von EE, Altman DG, Egger M, Pocock SJ, Gotzsche PC, Vandenbroucke JP. The Strengthening the Reporting of Observational Studies in Epidemiology (STROBE) statement: guidelines for reporting observational studies. Lancet 2007;147:573-7.

23. Da Costa BR, Cevallos M, Altman DG, et al. Uses and misuses of the STROBE statement: bibliographic study. BMJ Open 2011 26;1:e000048.

24. Oxford Centre for Triple Value Healthcare Ltd (3V) portfolio. The Critical Appraisals Skills Programme (CASP). Oxford Centre for Triple Value Healthcare Ltd (3V) portfolio 2020.

25. Padulo J, Oliva F, Frizziero A, Maffulli N. Muscles, Ligaments and Tendons Journal. Basic principles and recommendations in clinical and field Science Research: 2018 update. MLTJ 2018;8(3):305-7. 